\title{
Therapeutic approaches to gastroesophageal junction adenocarcinomas
}

\author{
Rachel L.G.M. Blom, ${ }^{1}$ Tatiana Bogush, ${ }^{2}$ Björn L.D.M. Brücher, ${ }^{3,4}$ Andrew C. Chang, ${ }^{5}$ \\ Mikhail Davydov, ${ }^{2}$ Evgeny Dudko, ${ }^{2}$ Trevor Leong, ${ }^{6}$ Boris Polotsky, ${ }^{2}$ Paul E. Swanson, ${ }^{7}$ \\ Peter S.N. van Rossum, ${ }^{8,9}$ Jelle P. Ruurda, ${ }^{8}$ Xavier Sagaert, ${ }^{10}$ Sergei Tjulandin, ${ }^{2}$ \\ Marie-Celine Schraepen, ${ }^{11}$ Meindert N. Sosef, ${ }^{11}$ and Richard van Hillegersberg ${ }^{8}$
}

${ }^{1}$ Department of Surgery, Onze Lieve Vrouwe Gasthuis, Amsterdam, the Netherlands. ${ }^{2}$ N.N. Blokhin Russian Cancer Research Center of the Russian Academy of Medical Sciences, Moscow, Russia. ${ }^{3}$ Surgical Oncology, Bon Secours Cancer Institute, Richmond, Virginia. ${ }^{4}$ Theodor-Billroth-Academy, Munich, Germany. ${ }^{5}$ Section of Thoracic Surgery, University of Michigan Health System, Ann Arbor, Michigan. ${ }^{6}$ Peter MacCallum Cancer Centre, Melbourne, Australia. ${ }^{7}$ University of Washington, Seattle, Washington. ${ }^{8}$ Department of Surgery, University Medical Center Utrecht, Utrecht, the Netherlands. ${ }^{9}$ Department of Radiotherapy, University Medical Center Utrecht, Utrecht, the Netherlands. ${ }^{10}$ Translational Cell \& Tissue Research, Department of Imaging and Pathology, University of KU Leuven, Leuven, Belgium. ${ }^{11}$ Department of Surgery, Atrium Medical Center, Heerlen, the Netherlands

Address for correspondence: annals@nyas.org

The following, from the 12th OESO World Conference: Cancers of the Esophagus, includes commentaries on the distinction between adenocarcinomas above, below, or within the gastroesophageal junction; combined modality therapy; tumor markers for use in personalized medicine; PET-CT and endoscopic biopsies in the evaluation of response to neoadjuvant chemoradiation therapy; a standardized grading system for tumor regression in squamous cell cancer and adenocarcinoma; the experimental basis for new approaches to medical treatment; the criteria measuring response in esophageal cancer; and the impact of novel imaging on staging and response assessment.

Keywords: gastroesophageal junction; PET-CT; tumor regression; combined modality therapy; tumor markers; tumor grading; OESO

\section{Concise summary}

A preponderance of data reported in the literature supports the use of multimodality therapy for the treatment gastroesophageal junction (GEJ) adenocarcinomas, albeit largely by extrapolation. When distinguished by tumor location, major therapeutic differences largely result from the extent of tumor resection, rather from any differences in neoadjuvant or adjuvant treatment regimens. Perioperative treatment, either chemotherapy or chemotherapy with concurrent radiation therapy, provided a statistically significant overall survival benefit compared to resection alone for esophagogastric carcinomas, with a hazard ratio of 0.81 favoring combined modality therapy. Although the benefit of any perioperative treatment regimen was less apparent for patients with esophageal cancers, with a hazard ratio of 0.87 , a cohort of patients, consisting predominantly of those with esophageal or GEJ carcinomas that had received preoperative chemoradiation, was found to have a survival benefit, with a hazard ratio of 0.70 . Perioperative treatment regimens predominantly include platinum-based chemotherapy, regardless of site of tumor origin, and are considered for more advanced-stage tumors. For Siewert type II or type III adenocarcinomas, perioperative chemotherapy without radiation therapy is typically recommended, whereas for more proximal tumors (type I or type II), concurrent radiation therapy can be administered preoperatively with carboplatin and paclitaxel.

There have been many randomized trials of preoperative chemoradiotherapy (CRT) versus surgery alone. The largest and most recent trial is the CROSS study that randomized patients with either 
squamous cell carcinoma (SCC) or adenocarcinoma to either surgery alone or preoperative CRT. The CRT regimen was extremely well tolerated, and although the postoperative complication rates were somewhat higher than expected, there were no significant differences between the two groups. The principal study supporting the use of preoperative chemotherapy for esophageal cancer is the Medical Research Council oestradiol (OE2) trial. With a median follow-up of 37 months, the 2-year survivals were $43 \%$ for preoperative chemotherapy versus $34 \%$ for surgery alone $(P=0.004)$. In a trial comparing CRT versus chemotherapy, because the study was closed early before reaching its target accrual, the improved survival with CRT did not reach statistical significance.

Currently recognized prognostic or predictive markers have clear evidentiary support for their use in the emerging practice of personalized medicine. In both esophageal adenocarcinoma (EAC) and squamous cell cancer, recent exomic and genomic sequencing analyses have strengthened the objective foundation for the importance of selected molecular pathways to the genesis and progression of disease. In adenocarcinoma, a variety of molecular pathways have been targeted for molecular analysis and clinical intervention. The most important in current practice is MAP kinase, associated with tyrosine kinase receptors and downstream effectors through KRAS and BRAF. The two most common are HER2 and EGFR. The importance of the pTEN tumor suppressor, through its inhibitory effects on the PI3K-Akt-mTOR pathway, has been independently established in a subset of EAC. The role of smallmolecule inhibitors in counteracting the molecular effects of epigenetic alterations in the canonical Wnt signaling pathway is also intriguing, when interventions for adenocarcinoma and squamous cell cancer are compared to those proposed for cancer models more dependent on mutational alterations in the pathway. Notch signaling is also potentially important to both adenocarcinoma and squamous cell cancer, with loss of SMAD4 inhibition seen in adenocarcinoma and loss of function due to mutation in squamous cell cancer. Several constraints, including the degree of heterogeneity for these markers within target populations and the as yet poorly understood nature of the interactions of different functional pathways on treatable foci, complicate the attempts to determine the relative impor- tance of a given biomarker to both risk assessment and patient management.

Early identification of pathological complete response (pCR) or pathological non-response (pNR) during or after neoadjuvant treatment is of clinical importance to reduce toxicity and improve personalized patient care. Negative predictive values of a test are of particular interest, as these refer to the likeliness of a suspected complete responsebased on the test result in an individual patientto truly reflect pCR. Current imaging lacks accurate assessment of response to neoadjuvant treatment in esophageal cancer. At the University Medical Center Utrecht (the Netherlands), the PRIOR trial (PReoperative Identification Of Response to neoadjuvant chemo-radiotherapy for esophageal cancer) is designed as an explorative single-center diagnostic study to assess both the distinct and combined value of advanced MRI and PET-CT scanning in the evaluation of treatment response to neoadjuvant CRT for patients with esophageal cancer.

No generally accepted standardized clinical or histopathological response evaluation system following treatment has been established so far. Tumor regression-grade analysis as a marker of response was undertaken in previous studies and scored from complete regression to absent regression. It remains a significant predictor of disease-free survival (DFS), although it suffers from high inter-observer variability. The three-tiered tumor regression scoring system seems to be more easily implemented, more reproducible, stronger, and clearer with regard to prognostic impact. It may provide highly valuable prognostic information, which may even exceed the prognostic impact of the current TNM classification.

The role of $\mathrm{ABC}$ transporters in the mechanism of multiple drug resistance (MDR) to most antitumor agents used in the clinical practice has been scientifically proven. The influence of cisplatin and carboplatin on intracellular accumulation of the model MDR drug doxorubicin in multiple drugresistant cells was studied using flow cytometry. Both drugs can enhance intracellular and intranuclear accumulation of the model drug doxorubicin. Both should be considered as effective antitumor agents and inhibitors of the mechanism of innate or induced resistance to drugs that are used in combination. Modification of cisplatin administration may improve response to chemotherapy, but it is 
obvious then that, to maximize platinum inhibition of ABC-transporter function, the sequence of drug administration "platinum $\rightarrow$ multiple drug resistance" should be maintained during the entire chemotherapy duration.

Clinical-response classification in use during and/or after neoadjuvant radio- and chemotherapy is limited by the lack of non-invasive techniques that allow differentiation between responders and non-responders. Clinical response by endoscopy, endoluminal ultrasound (EUS), and/or computed tomography (CT) cannot differentiate between viable tumor, inflammatory reaction, edema, or scar tissue, and, in case of endoscopy, it is also investigator dependent. There are not standardized criteria such as which positron emission tomography (PET) parameters recorded should be used. There is a lack of strict criteria as to which variables under investigation need to be included, and patient management trials using response as a judgment variable for determining different forms of multimodal treatment are still missing. Molecular biomarkers and findings in biogenomics should be used for collating information about responses to multimodal therapy.

Response assessment is needed to avoid ineffective neoadjuvant therapy, with the risk for patients to be exposed to negative consequences such as toxicity and delayed surgical therapy.

Fluorodeoxyglucose PET has been used as an accurate diagnostic method for early assessment of tumor response, but the negative predicting value is insufficient to apply PET scan for response assessment early in the course of neoadjuvant CRT. Diffusion-weighted magnetic resonance imaging (dMRI) might be an alternative with several advantages. Tumor hypoxia could be useful in the identification of patients who may benefit from CRT with specific antihypoxic treatments such as bioreductive drugs of hypoxic radiosensitizers and, in the future, a patient-tailored therapy with surgery on indication after completion of neoadjuvant CRT may be feasible.

\section{Is there any therapeutic reason to make distinctions in the site of origin for adenocarcinomas arising slightly above, slightly below, or at the GEJ?}

\author{
Andrew C. Chang \\ andrwchg@umich.edu
}

Adenocarcinomas arising either in the distal esophagus within $5 \mathrm{~cm}$ of the GEJ (Siewert type I) or at the GEJ (Siewert type II) are typically treated as esophageal carcinomas, whereas tumors arising in the gastric cardia within $5 \mathrm{~cm}$ of the GEJ (Siewert type III) are typically treated as gastric carcinomas. ${ }^{1}$ A preponderance of data reported in the literature supports the use of multimodality therapy for the treatment of GEJ adenocarcinomas, albeit largely by extrapolation. Major therapeutic differences, when distinguished by tumor location, largely result from the extent of tumor resection, rather than from any differences in neoadjuvant or adjuvant treatment regimens.

In deciding upon operative technique, whether esophagectomy, gastrectomy, or esophagogastrectomy, treatment planning should consider both the degree of proximal esophageal involvement and extent of lymphatic involvement. A proximal tumor- free esophageal margin of greater than $5 \mathrm{~cm}$, as measured in situ, has been associated with improved overall survival following resection, particularly for patients whose staging indicates six or fewer involved lymph nodes (N2). ${ }^{2}$ In a multi-institutional, international retrospective study of subjects undergoing esophageal resection alone for carcinoma, the number of resected lymph nodes appeared to be associated independently with improved overall survival. ${ }^{3}$ Furthermore, among patients found to have eight or more metastatic lymph nodes, nearly all developed systemic disease within 5 years of resection. ${ }^{4}$

In a recent Cochrane systematic review and meta-analysis, ${ }^{5}$ perioperative treatment, either chemotherapy or chemotherapy with concurrent radiation therapy, provided a statistically significant overall survival benefit compared to resection alone for esophagogastric carcinomas, with a hazard ratio (HR) of 0.81 (95\% CI 0.73-0.89) favoring combined modality therapy, as derived from 14 studies including 2422 subjects. For this analysis, individual patient data were obtained from relevant published clinical trials, providing the opportunity to focus specifically on adenocarcinoma. In sub-group analysis, patients with GEJ carcinomas (six studies, 470 
subjects) obtained a survival benefit with perioperative treatment, with HR 0.69 (0.54-0.87). Although the benefit of any perioperative treatment regimen was less apparent for patients with esophageal cancers, with HR 0.87 (0.73-1.05), a cohort of patients, consisting predominantly of those with esophageal or GEJ carcinomas that had received preoperative chemoradiation, was found to have a survival benefit, with HR 0.70 (0.50-0.99).

Perioperative treatment regimens predominantly include platinum-based chemotherapy, regardless of site of tumor origin, ${ }^{6}$ and are considered for more advanced stage tumors (stage IB, II or worse). For type II or type III AEGs, perioperative chemotherapy without radiation therapy is typically recommended, ${ }^{7}$ whereas for more proximal AEGs (Siewert type I or type II), concurrent radiation therapy can be administered preoperatively ${ }^{8}$ with carboplatin and paclitaxel.

\section{Combined modality therapy}

Trevor Leong

trevor.leong@petermac.org

Currently, the majority of patients with resectable esophageal cancer are managed with neoadjuvant therapy before surgical resection. With the exception of early stage T1-2, N0 disease, most patients with resectable tumors will be offered either preoperative CRT (preop CRT) or preoperative chemotherapy (preop CT) alone.

\section{Preop CRT}

There have been many randomized trials of preop CRT versus surgery alone. These trials have utilized a wide variety of radiotherapy dose/fractionation regimens. Most have employed cisplatin and 5-fluorouracil (5-FU) as the chemotherapy backbone. Because these individual trials have shown conflicting results, several meta-analyses have been undertaken to evaluate the utility of preop CRT. The Australasian Gastro-Intestinal Trials Group (AGITG) meta-analysis, which includes data from 13 randomized trials, showed a $22 \%$ reduction in allcause mortality for preop CRT, which corresponds to an absolute survival benefit at 2 years of $8.7 \% .{ }^{9}$ The survival benefits for preop CRT were slightly higher for adenocarcinoma than for SCC, with hazard ratios of 0.75 and 0.80 , respectively. The first study to show a benefit for preop CRT was reported by Walsh et al. in $1996 .{ }^{10}$ This trial ran- domized patients with EAC only to either surgery alone or preop CRT consisting of 5-FU/cisplatin combined with 40 Gy of radiation. Although this study showed improved survival with preop CRT, one of the major criticisms of the trial has been that survival in the surgery-alone group was very poor, with only $6 \%$ of patients alive at 3 years. The Cancer and Leukemia Group B (CALGB) study from the United States is interesting because it is the only one that has employed a radical radiation dose of 50.4 Gy. ${ }^{11}$ Although this trial closed early before reaching its target accrual, it nevertheless showed a survival benefit for preop CRT. Despite the use of high radiation doses, the surgical complication rates were similar between the two groups. The largest and most recent trial is the Chemoradiotherapy for Oesophageal Cancer followed by Surgery Study CROSS study from the Netherlands that randomized 368 patients with either SCC or adenocarcinoma to either surgery alone or preop CRT. ${ }^{8}$ The chemoradiation regimen consisted of weekly carboplatin and paclitaxel combined with 41.4 Gy of radiation. The 5 -year survival rates were $47 \%$ for preop CRT versus $34 \%$ for surgery alone $(P=0.003)$. The chemoradiation regimen was extremely well tolerated, and although the postoperative complication rates were somewhat higher than expected, there were no significant differences between the two groups.

\section{Preop CT}

There have been nine randomized trials of preop CT versus surgery alone. The AGITG meta-analysis also evaluated the utility of preop CT, and found improvements when compared to surgery alone, although the treatment effects were not as large as for preop CRT. ${ }^{9}$ There was a $13 \%$ reduction in all-cause mortality for preop CT, which corresponds to an absolute survival benefit at 2 years of $5.1 \%$. A subgroup analysis by histological subtype showed that the benefit was statistically significant for adenocarcinoma but not for SCC. The principal study supporting the use of preop CT for esophageal cancer is the Medical Research Council Oesophageal Cancer Working Group trial (OE2). ${ }^{12}$ This trial randomized 802 patients with either SCC or adenocarcinoma to either surgery alone or preop CT consisting of two cycles of 5-FU/cisplatin. With a median follow-up of 37 months, the 2 year survivals were $43 \%$ for preop CT versus $34 \%$ for surgery alone $(P=0.004)$. 


\section{Preop CRT versus preop CT}

Which neoadjuvant treatment is superior: preop CRT or preop CT? A study reported by Stahl et al. has attempted to answer this question. ${ }^{13}$ This trial randomized 119 patients with adenocarcinoma of the GEJ to either preop CRT or preop CT. Both groups received induction chemotherapy with cisplatin/5FU/leucovorin. Patients in the CRT group then received 30 Gy of radiation with concurrent cisplatin/etoposide. Although the trial was planned to recruit 354 patients, it was closed early due to poor accrual. With a median follow-up of 46 months, the 3-year survivals were $47 \%$ for preop CRT versus $28 \%$ for preop CT. However, because the study was closed early before reaching its target accrual, the improved survival with CRT did not reach statistical significance $(P=0.07)$. The pathological complete response rate was significantly increased from $2 \%$ for preop CT to $16 \%$ for preop CRT $(P=0.03)$. The AGITG meta-analysis also compared the survival benefits of preop CRT versus preop CT. ${ }^{9}$ The hazard ratio for all-cause mortality was 0.88 in favor of preop CRT, but again this did not reach statistical significance $(P=0.07)$. In summary, there are two alternative standards of care for neoadjuvant therapy in esophageal cancer; preop CRT, which is used mainly in North America, and preop CT, which is used mainly in the United Kingdom. Ongoing studies are evaluating the role of PET and molecular biomarkers to predict response to neoadjuvant treatment.

\section{Tumor markers for use in personalized medicine}

Paul E. Swanson

paul.swanson@cls.ab.ca

The complexity of molecular pathways in human neoplasms is astounding, and the potential role of mutational, epigenetic, or other molecular alterations in these pathways as biomarkers for diagnosis, prognosis, and prediction of global and targeted therapeutic interventions is similarly complex. A surprising number of these receptor and pathway effector molecules already have both specific antibody and small-molecule inhibitor pharmacologic agents ready for preclinical evaluation and immediate clinical applications. Yet, few currently recognized prognostic or predictive markers have clear evidentiary support for their use in the emerging practice of personalized medicine. ${ }^{14}$ The challenge rests in identifying specific molecular markers that are reproducibly associated with disease progression or that serve as robust predictors of treatment response. Dissecting molecular pathways to identify common genetic or chromosomal alterations provides a reservoir of markers of interest, but translating these markers into safe and effective clinical interventions remains elusive.

In both EAC and SCC, recent exomic and genomic sequencing analyses have strengthened the objective foundation for the importance of selected molecular pathways to the genesis and progression of disease. ${ }^{15,16}$ These studies also reiterate that the repertoires of abnormalities in critical genes and gene pathways differ substantially between diagnostic groups, while emphasizing that multiple pathways may be altered within diagnostic groups. This intrinsic heterogeneity makes it likely that even commonly encountered abnormalities will only be relevant to the treatment of selected populations of EAC and SCC patients and that combinations of biomarkers and therapeutic targets will have the greatest impact on management of a given patient or patient cohort. With that in mind, it will be necessary to understand interactions between pathways to fully appreciate the predictive value of selected markers.

\section{Pathways and markers of interest}

In EAC, a variety of molecular pathways have been targeted for molecular analysis and clinical intervention. Perhaps the most important of these in current practice is MAP kinase, associated with tyrosine kinase receptors and downstream effectors through KRAS and BRAF. The two most common targets (used now in esophageal neoplasms after their potential utility was established in other sites) are HER2 and EGFR. ${ }^{16-19}$ HER2 is of interest because amplification of this genetic locus (Fig. 1) is associated with overexpression of the functional receptor, establishing it as a target for intervention, while both EGFR mutation and amplification may be of significance in EAC and SCC. The ToGA trial, ${ }^{18}$ together with preliminary studies of esophageal neoplasms, suggest that the expected positive rate for HER 2 amplification in gastric, GEJ, and EAC is no greater than $20-25 \%$, and that the clinical response to combined anti-HER2 and chemotherapy in HER2-amplified 


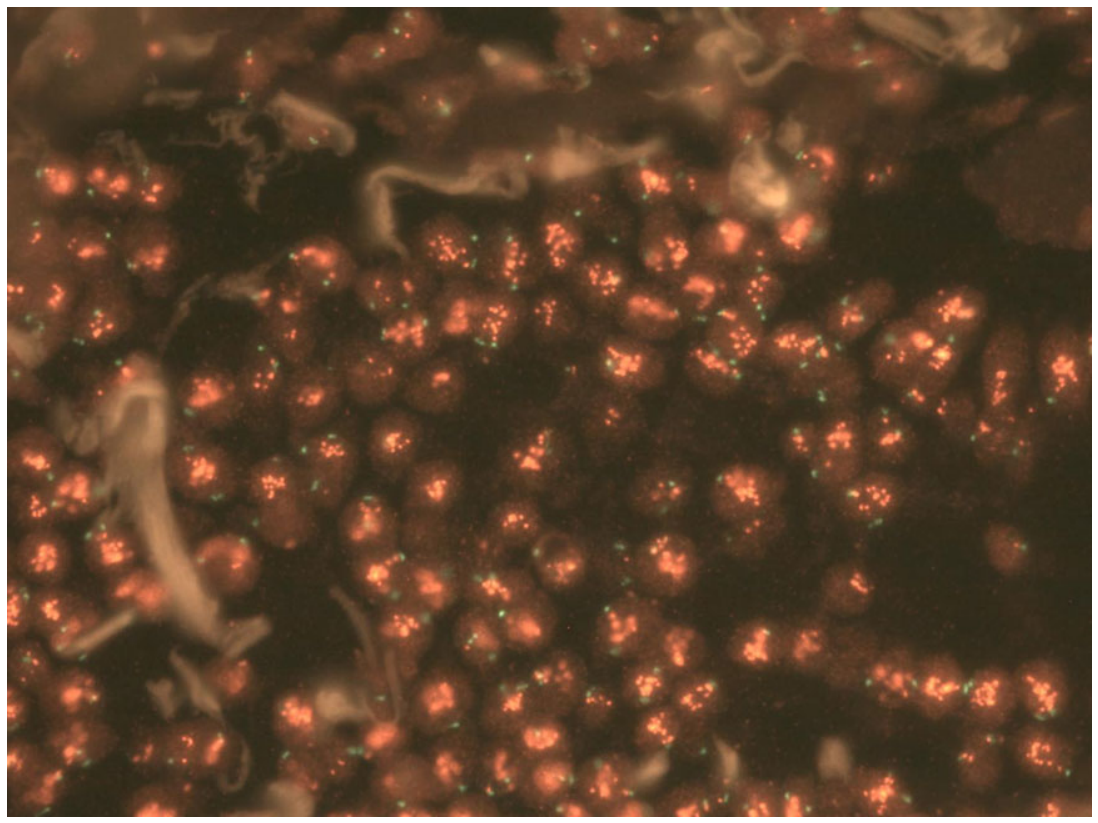

Figure 1. Demonstration of HER2 gene amplification in esophageal adenocarcinoma by fluorescent in situ hybridization. In this image, orange signals identify copies of the HER2 gene on chromosome 17, while the green signals identify centromeric elements of this chromosome, using the Cep17 probe. The Her2:Cep17 ratio in this case is 5.9 (Her2:Cep17 fluorescent in situ hybridization; $600 \times)$.

neoplasms is significant. Interestingly, this predictive role for HER2 is not accompanied by a similar value in risk stratification, since neither HER2 nor EGFR status in EAC reliably predicts prognosis independent of anticipated treatment effect. Unfortunately, adverse clinical effects of antibody-based treatments to these receptor moieties-especially EGFR-are potentially limiting in both EAC and SCC. ${ }^{17}$

The importance of the pTEN tumor suppressor, through its inhibitory effects on the PI3K-AktmTOR pathway, has been independently established in a subset of EAC. ${ }^{19}$ However, because its clinical significance relies on loss of function through mutation or epigenetic alteration, attention shifts to downstream elements of the pathway, where (phospho)Akt and mTOR emerge as more interesting therapeutic targets. pTEN also serves as a model for interactions across pathways that mitigate (or potentially augment) the clinical effectiveness of targeted therapies. A recent analysis by Eto et al. demonstrated that the microRNA-21-pTEN pathway selectively regulates tumor responsiveness to trastuzumab in gastric carcinoma, ${ }^{20}$ an observation that has not yet been extended to EAC.
The role of small-molecule inhibitors in counteracting the molecular effects of epigenetic alterations in the canonical Wnt signaling pathway is also intriguing, when interventions for EAC and SCC are compared to those proposed for cancer models more dependent on mutational alterations in the pathway (such as colorectal carcinoma). Chemotherapies targeting EAC and SCC populations identified as MSIhigh-often due to epigenetic effects (primarily CpG-island methylation of the upstream promoters of mismatch-repair genes) and more common in SCC than $\mathrm{EAC}^{21}$ —are also relevant to modern oncologic management.

Markers that stratify risk for tumor progression include (but are clearly not limited to) VEGF-C/VEGFR ${ }^{14-17,22}$ (in both EAC and SCC), TP53, NF- $\kappa$ B, TGF- $\beta / S M A D 4$, RUNX3, and GATA6/BMP4, the latter five best illustrated in BEassociated dysplasia and EAC. ${ }^{14}$ Notch signaling is also potentially important to both EAC and SCC, with loss of SMAD4 inhibition seen in $\mathrm{EAC}^{16}$ and loss of function due to mutation in SCC. ${ }^{15} \mathrm{Fi}^{-}$ nally, novel and potentially useful biomarkers associated with progression from BE dysplasia to EAC have been identified in large-scale genomic ${ }^{15,16}$ and 
serum glycomic analyses. ${ }^{23}$ These include the Rac pathway proteins ELMO1 and DOCK2 (implicated in cell motility and stromal invasion) $;{ }^{15}$ fetuin $\mathrm{B}$, a cystatin modestly upregulated in BE-HGD (highgrade dysplasia) and significantly increased in EAC; and elastin microfibril interface located protein 2 (EMILIN-2), an extracellular matrix glycoprotein that is involved in activation of the extrinsic pathway of apoptosis, inhibits cell growth, and is increased in HGD and EAC relative to disease-free controls. ${ }^{23}$

\section{Conclusions}

Analyses of genetic and epigenetic alterations in EAC and esophageal SSC and their precursor lesions have identified a number of possible targets for treatment. However, several constraints, including the degree of heterogeneity for these markers within target populations and the as yet poorly understood nature of the interactions of different functional pathways on treatable foci, complicate-even obfuscate-our attempts to determine the relative importance of a given biomarker to both risk assessment and patient management. Nonetheless, the yield from increasingly powerful techniques available for novel marker discovery drive optimism that the reality of individualized targeted therapy (personalized medicine) may begin to approach the anticipated potential of patient management in the era of molecular medicine.

\section{Evaluation of response to neoadjuvant chemoradiotherapy in esophageal cancer using multimodality imaging}

Peter S.N. van Rossum, Richard van Hillegersberg, and Jelle P. Ruurda

P.S.N.vanRossum2-@umcutrecht.nl

CRT followed by surgery has become the current standard of treatment for patients with resectable esophageal cancer. ${ }^{8}$ This regimen has increased the percentage of microscopic radical resections up to $92 \%$, as opposed to $69 \%$ in the surgery-only control group. Furthermore, the 5-year survival and median survival improved by $13 \%$ and 25.4 months, respectively. Interestingly, a pCR was found in $29 \%$ of neoadjuvantly treated patients. This absence of residual cancer in the resection specimen is an important independent prognostic factor, and it has been speculated that surgery might be omitted in this selected group of patients in the future. On the contrary, approximately $20 \%$ of patients in the CRT arm of the trial showed a pNR. These patients suffer from ineffective toxic CRT, and probably surgery is unnecessarily delayed. Early identification of pCR or pNR during or after neoadjuvant treatment is of clinical importance to reduce toxicity and improve personalized patient care.

Multiple studies describe the value of different imaging modalities in the evaluation of response to neoadjuvant treatment as compared to the gold standard of histopathological tumor regression. With regard to this matter, negative predictive values (NPVs) of a test are of particular interest, as these refer to the likeliness of a suspected complete response-based on the test result in an individual patient-to truly reflect pCR. With pre- and post-treatment endoscopies, poor NPVs of $31-44 \%$ are found, and these do not significantly improve by adding biopsies. ${ }^{24,25}$ The underlying submucosal extent of the residual tumor can be masked by a normal-appearing lumen, and sampling errors occur as deep and spread residual foci as well as remaining metastatic lymph nodes that can be easily missed. Furthermore, in cases of residual disease endoscopy with or without biopsies, it is not possible to distinguish non-responders from partial responders. Other studies looked into one- or twodimensional size measurements using EUS. Disappointingly, reported NPVs varied from $17 \%$ to $69 \%{ }^{25-27}$ The main limitation of EUS is overstaging attributable to the difficulty of differentiating fibrosis and inflammation from residual tumor. Furthermore, EUS is an invasive technique, with a known failure rate of $13-25 \%$ due to stenotic tumors.

Studies on repeated CT for response evaluation were pooled in a systematic review that reported a maximum joint sensitivity and specificity of only $54 \% .{ }^{28}$ In a 3-D CT study, the tumor volume in responders was found to paradoxically increase over time owing to inflammation and fibrosis, implying that dimensional criteria alone cannot be trusted for response evaluation. ${ }^{29}$ Therefore, metabolic assessment rather than spatial assessment may be helpful. In the past decade, PET and combined PET-CT studies were performed for this reason. A systematic review and meta-analysis reveal a pooled sensitivity and specificity of around $70 \%{ }^{30}$ Available PET studies are heterogeneous and mostly single center and there is a need for standardization of protocols and well-designed multicenter trials. New developments that could potentially improve the value of 
PET-CT include combining spatial and metabolic information and using computer-based comprehensive spatial-temporal features.

Since all currently studied modalities yield unsatisfactory results in the assessment of response to neoadjuvant treatment in esophageal cancer so far, there might be a need for a new, more accurate tool. Diffusion-weighted imaging (DWI) is a functional MRI technique that derives its image contrast from differences in the motion of water molecules between tissues. Tumor tissue shows low free-water diffusion owing to the high cellularity and intact cell membranes. But as tumor cells die from treatment (e.g., show a response), cellularity decreases, resulting in an increase of free diffusion of water molecules. In rectal cancer, it was already shown that pathological complete responders tend to have a high increase of diffusion over time. ${ }^{31}$ Although this relatively new modality has been proven to be exceptionally valuable for treatment-response assessment in rectal cancer, its application in esophageal cancer has hardly been explored. ${ }^{32}$ Therefore, at the University Medical Center Utrecht (the Netherlands) the PRIOR trial was designed as an explorative singlecenter diagnostic study to assess both the distinct and combined value of advanced MRI and PET-CT scanning in the evaluation of treatment response to neoadjuvant CRT for patients with esophageal cancer.

In the past year, in Utrecht, an MRI-optimization study was conducted in 20 patients in order to optimize scan parameters for both anatomical and functional MRI sequences including T1/T2-weighted imaging, DWI, and dynamic contrast-enhanced MRI (DCE-MRI). Imaging quality, protocol robustness, and reproducibility were optimized and geometrical disturbances and motion artifacts were reduced. So far, preliminary results from pre- and post-treatment DWI scans and histopathological assessment of tumor regression indicate promising results. The scan parameters derived from this optimization study will be used in the actual treatment response study: the PRIOR trial, which is currently ongoing. In the PRIOR trial, 30 patients with resectable esophageal cancer receiving neoadjuvant CRT before surgery will undergo advanced MRI and PET-CT scans before, during, and after neoadjuvant treatment. All images will be co-registered using sophisticated deformable image-registration algorithms, allowing voxel-by-voxel analysis of all image modalities over all three time points for each patient. Imaging response measurements will be compared with the histopathological tumor regression in the resection specimen as the gold standard.

In conclusion, current imaging lacks accurate assessment of response to neoadjuvant treatment in esophageal cancer. PET-CT seems the best modality so far, while MRI is a promising new tool, as suggested by rectal cancer studies and our preliminary experience in esophageal cancer. The PRIOR trial will be the first study worldwide to explore the distinct and combined value of advanced anatomical and functional MRI and PET-CT techniques for this purpose. Ultimately, machine-learning techniques will be applied to develop a multiparametric model that accurately model that accurately predicts response based on multimodality imaging.

\section{Is there a standardized grading system for tumor regression in SCC and in adenocarcinoma?}

\section{Xavier Sagaert \\ xavier.sagaert@uzleuven.be}

Esophageal cancer is the 8th most common cancer worldwide, and more than $80 \%$ of cancers occur in less developed countries. There are various subtypes, primarily SCC (approximately 90-95\% of all esophageal cancer worldwide) and EAC (approximately $50-80 \%$ of all esophageal cancer in the Western world). Esophageal squamous cell cancer mainly occurs in the so called "Asian belt" and arises from the non-keratinizing stratified squamous epithelium that lines the esophagus. Adenocarcinomas of the esophagus and the GEJ are the most dramatically rising cancers in the Western world and arise from a single layer of columnar, usually mucussecreting, epithelium that is present at the junction of the esophagus and stomach (a process called Barrett's metaplasia). Importantly, SCCs are associated with heavy tobacco and alcohol consumption, while adenocarcinomas are often associated with a history of gastroesophageal reflux disease (GERD) and Barrett's esophagus (BE).

To improve the outcome of this malignancy with an already very bad overall prognosis, esophageal cancers infiltrating beyond the mucosa are mostly treated by neo-adjuvant CRT before invasive and extensive surgery. ${ }^{7,33}$ Remarkably, while $20 \%$ of patients will reach a complete pathological 
complete response after CRT, in about $30-50 \%$ of patients no measurable response will be achieved at all. ${ }^{34}$ While a predictor of response/resistance to CRT at diagnosis (that could save responding patients from invasive surgery and avoid the toxicity of unnecessary CRT and delay of surgery in nonresponding patients) has not been identified yet, it is generally believed that histological tumor regression after chemotherapy is an important objective parameter that has prognostic value in several studies.

The prognostic significance of the histopathological response to cytotoxic therapy in esophageal cancer specimens (predominantly SCC) was first reported by Mandard et al. ${ }^{35}$ Tumor regression grade (TRG) analysis, as a marker of treatment response, was undertaken in that study and scored from complete regression (TRG1) to absent regression (TRG5). In brief, TRG1 (complete regression) showed absence of residual cancer and fibrosis extending through the different layers of the esophageal wall; TRG2 was characterized by the presence of rare residual cancer cells scattered through the fibrosis; TRG3 was characterized by an increase in the number of residual cancer cells, but fibrosis predominated; TRG4 showed residual cancer outgrowing fibrosis; and TRG5 was characterized by the absence of regressive changes. Of the 93 cases in the study, 42\% were TRG1-2, 20\% TRG3, and $33 \%$ were TRG4-5. Tumor size, pathological lymph node status, TRG, and esophageal wall involvement correlated highly with DFS. On multivariate analysis, however, only TRG (TRG1-3 versus TRG4-5) remained a significant $(P<0.001)$ predictor of DFS. However, while the Mandard TRG is highly prognostic and remains universally the most widely used regression system in esophageal cancer, it has not been validated in adenocarcinomas and has been shown to suffer from high interobserver variability. Hence, other grading scoring systems have been introduced, although they are not widely used. Of particular interest, Langer et al. applied a three-score tumor regression system for EAC that was originally introduced for gastric cancer (albeit slightly modified), similar to a score that they also successfully applied to esophageal squamous carcinomas and rectal cancer. ${ }^{36}$ These threetiered regression scoring systems have also been used for larger retrospective studies by others, as they seem to be more easily implemented, more reproducible, stronger, and clearer with regard to prog- nostic impact. ${ }^{37,38}$ Langer et al. even demonstrated that these three-tiered histological TRG systems that score the effects of preoperative chemotherapy in esophageal cancer provided highly valuable prognostic information, which may even exceed the prognostic impact of the current TNM classification of these tumors.

In conclusion, despite tons of data and its prognostic significance, no generally accepted standardized clinical or histopathological response evaluation system has been established so far. Nevertheless, it is strongly recommended that there be implementation of a standardized tumor regression-grading system in pathological reports of EAC treated by neoadjuvant chemotherapy.

\section{Experimental and clinical basis for optimization of cisplatin administration schedule to improve chemotherapy efficacy in patients with locally advanced esophageal cancer}

Tatiana Bogush, Evgeny Dudko, Boris Polotsky, Sergei Tjulandin, and Mikhail Davydov labmedchem@mail.ru

\section{Background}

It is commonly accepted that chemotherapy has unsatisfactory effects in patients with esophageal cancer, and approaches to increase treatment efficacy are developed intensively. Studies investigating this problem demonstrated that MDR phenotype, associated with expression of energy-dependent $A B C$ transporters extruding MDR drugs out of the cells, was found in most esophageal cancer cases. ${ }^{39-41}$ At the same time, the role of $\mathrm{ABC}$ transporters in the mechanism of multiple drug resistance to most antitumor agents used in the clinical practice has been scientifically proven. The idea of studying platinum drugs as polyvalent MDR inhibitors acting on various $\mathrm{ABC}$ transporters' functioning emerged in attempts to determine why MDR tumors responded to MDR cytostatics in combination with platinum derivatives. This clinical fact is well documented in the treatment of cancers of different sites, including esophageal tumors.

We have tried to explain this phenomenon on the basis of platinum properties described in the literature (i.e., binding to ATP that is needed for energydependent $\mathrm{ABC}$ transporters to function and to bind to various proteins). ${ }^{42}$ If this is true, then it is logical to assume that platinum agents inhibit $A B C$ 
transporter function and, as a result, overcome or reduce MDR to MDR agents used in combination with platinum. Direct experiments proved this assumption true.

\section{Experimental investigation}

The influence of cisplatin and carboplatin on intracellular accumulation of the model MDR drug doxorubicin in MDR cells was studied using flow cytometry. ${ }^{43}$ It was demonstrated that the platinum derivatives inhibited $\mathrm{ABC}$ transporter function. ${ }^{44}$ Both drugs can enhance intracellular and, what is still more important, intranuclear accumulation of the model MDR drug doxorubicin. This means that cisplatin and carboplatin should both be considered effective antitumor agents and inhibitors of the mechanism of innate or induced resistance to MDR drugs that are used in combination with them. In fact, this is an explanation of the abovementioned phenomenon of increased efficacy of platinum agents in combination with inactive MDR cytostatics in treatment of esophageal cancer. Our own clinical experience may be a positive example demonstrating the efficacy of this approach.

\section{Clinical pilot investigation}

Design. Thirty-six patients with locally advanced esophageal cancer received preoperative chemotherapy with cisplatin, etoposide, 5-FU, leukovorin (FLEP); cisplatin being administered by standard schedule with cisplatin administered on day 1 , or by a modified schedule, with cisplatin given on days $1-3$. The remaining drugs were always given on a daily basis, with etoposide administered in modified schedule after cisplatin. The two treatment arms were well balanced by main clinical characteristics. Response was assessed after two 3-day cycles with a 3-week interval.

Results. The number of cases demonstrating decreased severity of dysphagia after completion of chemotherapy was greater in the modified-schedule group ( $43 \%$ vs. $24 \%$ of cases), although there were more patients with higher dysphagia intensity in this group at baseline ( $19 \%$ vs. no patients with grade III dysphagia). More patients receiving a modified FLEP regimen as compared to the standard regimen group demonstrated decrease ( $60 \%$ vs. $48 \%$ ) or no change ( $40 \%$ vs. $26 \%$ ) in disease extent by $x$-ray after chemotherapy completion. Complete responses ( $20 \%$ of cases) were shown in the modified-regimen group only, and no patients in that group had progressive disease, versus $26 \%$ of cases with progressive disease in the standard-regimen group. And finally, more patients receiving modified FLEP survived 1 year of follow-up (79\% vs. $65 \%$ ).

Conclusion. Although these findings are but interim, we nevertheless believe that modification of cisplatin-administration schedule alone may improve response to chemotherapy with MDR drugs in the MDR-resistant patient category because platinum drugs are inhibitors of the mechanism of innate or induced MDR. In particular, the results demonstrate proof in the principal efficacy of this approach on the example of the modified schedule of FLEP chemotherapy in patients with locally advanced esophageal cancer.

It is obvious, then, that to maximize platinum inhibition of $\mathrm{ABC}$-transporter function, the sequence of drug administration "platinum $\rightarrow$ MDR drugs" should be maintained during the entire chemotherapy duration. It is not always so in routine clinical practice, because sequence of administration of platinum derivatives and other cytostatics is not strongly established. We consider this detail to be of much importance, and think that it should be controlled in the clinical practice to ensure maximum therapeutic effect of MDR drugs in combination with platinum derivatives in the treatment of MDR-tumors including esophageal cancer.

[Supported by Russian Foundation for Basic Research Grants (No.13-04-01004-a, No. 12-0400028-a) and Grant of the President of the Russian Federation (No. 376.2012.4).]

\section{Are criteria measuring response in esophageal cancer appropriate?}

Björn L.D.M. Brücher

bbruecher-@gmx.de

\section{Introduction}

The actual response criteria have recently been reviewed. ${ }^{45}$ These criteria have been in use since 1981 and, according to these criteria, response to anticancer treatment has been defined as being a therapy-induced change of two perpendicular parameters in size by at least $50 \% .{ }^{46}$ One of the first suggestions that diameters be used for measuring response was proposed in $1966,{ }^{47}$ with the subsequent experiment of Moertel and Hanley in 1976: ${ }^{48} 16 \mathrm{ex}-$ perienced oncologists received different sizes of solid 
wooden spheres covered with a layer of rubber foam and were tasked with judging the change in size. An error of $50 \%$ resulted in $6.8 \%$ false positive, ${ }^{48}$ which in turn resulted in the recommendation that only complete or partial response should be used in the determination of the response to anticancer therapy. ${ }^{49}$ Subsequently, a 2-D measurement of one dimension was replaced by the decrease of the largest tumor diameter by $30 \%$, interpreting this as equivalent to a $50 \%$ decrease for a spherical lesion. ${ }^{50}$ As during the 1970s no standardized way of describing and/or judging response was available, the primary goal was necessary.

It has been shown that clinical response by endoscopy, EUS, and/or CT cannot differentiate between viable tumor, inflammatory reaction, edema, or scar tissue and, in case of endoscopy, it is also investigator dependent. ${ }^{51}$ Until now, histopathological response assessing the percentage rate of residual tumor cells has minimized potential errors owing to the known post-therapeutic heterogeneity. ${ }^{52}$ Newer methodological approaches include the use of fluordeoxyglucose PET (FDG-PET). ${ }^{28,53}$ However, a major challenge is that no standardized criteria such as which PET parameters recorded should be used: (e.g., difference of a PET investigation before and after a multimodal approach $(\triangle \mathrm{SUV}), \mathrm{SUV}_{\text {mean }}$ or $S U V_{\max }$, or even a $\Delta S U V_{\max }$ ). Another point of interest is that journals do not demand strict criteria as to which variables under investigation need to be included, and some groups provide all necessary data including sensitivity, specificity, positive, and negative predictive value (PPV, NPV), and overall accuracy, including 95\% confidence intervals (CI), while others do not. ${ }^{53}$ This is further complicated by the fact that there is no regulation in terms of different inclusion criteria: different histological entities (different tumor biology, ways of metastasis, and prognosis), different surgical approaches (different postoperative outcomes), differences in delay of response measurement, or difference in training and standardization of histopathological workup. Also, the increase in survival during implementation of neoadjuvant trials was performed in an area in which the operative procedures were standardized. This argument was clearly shown by the leading upper GI centers in the 1990s, revealing that the outcomes of patients treated during the 1980s was statistically significantly worse than that obtained in the 1990s. ${ }^{53}$ Furthermore, patient management trials using response as a judgement variable for determining different forms of multimodal treatment are still missing.

Although these factors have not been resolved to date, groups and companies have already declared that molecular biomarkers and findings in biogenomics should be used for collating information about responses to multimodal therapy. To date, no group has been able to provide the necessary scientific information for judging these biomarkers in terms of the half-life time of each biomarker, its daily changes in concentrations/activities, or how the determination should be standardized. Additionally, basic findings in biogenomics are also missing, which could serve as a standard for being included within a new response classification. Comparing tumors with different anatomy, embryology, and histology that reveal different tumor biologies makes the task of standardization even more complicated.

In upper-GI cancer, clinical response classification in use during and/or after neoadjuvant radio- and chemotherapy is limited by the lack of non-invasive techniques that allow differentiation between responders and non-responders. Additionally, the classification system for evaluating response needs to be revisited.

\section{The impact of novel imaging on staging and response assessment}

Marie-Celine Schraepen, Rachel L.G.M. Blom, Meindert N. Sosef

meindert.sosef@me.com

\section{Introduction}

Esophageal cancer is the sixth leading cause of cancer death worldwide, with 500,000 new cases each year. Survival rates for both M0 and M1 esophageal cancer in the Netherlands have shown steady improvements over recent decades. ${ }^{54}$ This may be due to centralization, resulting in improved patient selection, perioperative care, and surgical experience. A recent development is the introduction of neoadjuvant chemoradiation, which has led to an improved oncological outcome and an increased overall survival rate compared to patients who underwent surgery alone. ${ }^{55}$

\section{Staging is needed to avoid palliative surgery}

Esophageal cancer is known for its early dissemination. On diagnosis, at least half of the patients have 
locally advanced or metastatic disease. According to the American Joint Committee on Cancer (AJCC), $\mathrm{CT}$, PET, and integrated PET-CT are included in the staging techniques to establish the M-stage in esophageal cancer. ${ }^{56}$

Metastatic disease after completion of neoadjuvant CRT implies that curative treatment is no longer possible; thus, accurate patient selection is crucial. Several studies have described post-neoadjuvant therapy metastases in $8-17 \%$ of patients. ${ }^{57,58}$ Routine PET-CT after completion of the neoadjuvant therapy is recommended in the prevention of palliative resections. ${ }^{56}$

\section{Response assessment is needed to avoid ineffective neoadjuvant therapy}

As previously mentioned, neoadjuvant CRT before surgery can improve the oncological outcome of patients; however, not all patients show a response to neoadjuvant therapy. Non-responders do not benefit from neoadjuvant CRT but are exposed to the negative consequences such as toxicity and delayed surgical therapy.

FDG-PET has been used as an accurate diagnostic method for early assessment of tumor response in malignant lymphoma and non-small cell lung cancer to CRT. ${ }^{59}$ In esophageal cancer patients, a decrease in SUV, measured by FDG-PET before and 14 days after the start of CRT, was associated with a histopathological response. The negative predicting value was insufficient to apply FDG-PET for response assessment early in the course of neoadjuvant CRT. ${ }^{59}$

Diffusion-weighted magnetic resonance imaging (DW-MRI) might be an alternative to FDGPET. DW-MRI has several advantages compared to FDG-PET. Before DW-MRI is performed, fasting is not necessary; furthermore, no exogenous contrast material is required. However, preliminary results showed no correlation between pretreatment tumor apparent diffusion coefficient (ADC) and change in tumor ADC 10 days after the start of chemoradiotherapy. ${ }^{60}$

Tumor hypoxia is an important marker of cancer prognosis, because of its association with aggressive growth, resistance to anticancer therapy, and metastases. ${ }^{61}$ Therefore, tumor hypoxia could be useful in the identification of patients who may benefit from CRT with specific antihypoxic treatments such as bioreductive drugs of hypoxic radiosensitizers. ${ }^{62}$ Quantification of tumor hypoxia can be calculated by performing non-invasive PET imaging with hypoxia-specific 2-nitroimidazoles. This type of imaging needs further investigation to determine optimal imaging conditions. ${ }^{62}$

\section{Surgery on indication}

In the future, a patient-tailored therapy with surgery on indication after completion of neoadjuvant CRT may be feasible. Patients will need to undergo close surveillance after completion of neoadjuvant therapy. Only patients in whom a locoregional recurrence is highly suspected or proven, without any signs of distant dissemination, will be offered a surgical resection. ${ }^{63}$

\section{Conclusion}

In esophageal cancer treatment, neoadjuvant chemotherapy leads to an increase in overall survival. ${ }^{55}$ Accurate patient selection is crucial for a curative treatment. Response assessment in esophageal cancer is promising but needs to be further investigated before it can be implemented.

\section{Conflicts of interest}

The authors declare no conflicts of interest.

\section{References}

1. Marsman, W.A., G.N. Tytgat, F.J. ten Kate \& J.J.van Lanschot. 2005. Differences and similarities of adenocarcinomas of the esophagus and esophagogastric junction. J. Surg. Oncol. 92: $160-168$.

2. Barbour, A.P., N.P. Rizk, M. Gonen, et al. 2007. Adenocarcinoma of the gastroesophageal junction: influence of esophageal resection margin and operative approach on outcome. Ann. Surg. 246: 1-8.

3. Peyre, C.G., J.A. Hagen, S.R. DeMeester, et al. 2008. The number of lymph nodes removed predicts survival in esophageal cancer: an international study on the impact of extent of surgical resection. Ann. Surg. 248: 549-556.

4. Peyre, C.G., J.A. Hagen, S.R. DeMeeste, et al. 2008. Predicting systemic disease in patients with esophageal cancer after esophagectomy: a multinational study on the significance of the number of involved lymph nodes. Ann. Surg. 248: 979-985.

5. Ronellenfitsch, U., M. Schwarzbach, R. Hofheinz, et al. 2013. Perioperative chemo(radio)therapy versus primary surgery for resectable adenocarcinoma of the stomach, gastroesophageal junction, and lower esophagus. Cochrane Database Syst. Rev. 5: CD008107.

6. Apisarnthanarax, S. \& J.E. Tepper. 2008. Crossroads in the combined-modality management of gastroesophageal junction carcinomas. Gastrointest. Cancer Res. 2: 235-243.

7. Cunningham, D., W.H. Allum, S.P. Stenning, et al. 2006. Perioperative Chemotherapy versus Surgery Alone for 
Resectable Gastroesophageal Cancer. N. Engl. J. Med. 355: 11-20.

8. van Hagen, P., M.C. Hulshof, J.J. van Lanschot, et al. 2012. Preoperative chemoradiotherapy for esophageal or junctional cancer. N. Engl. J. Med. 366: 2074-2084.

9. Sjoquist, K.M., B.H. Burmeister, B.M. Smithers, et al. 2011. Survival after neoadjuvant chemotherapy or chemoradiotherapy for resectable oesophageal carcinoma: an updated meta-analysis. Lancet Oncol. 12: 681-692.

10. Walsh, T.N., N. Noonan, D. Hollywood, et al. 1996. A comparison of multimodal therapy and surgery for esophageal adenocarcinoma. N. Engl. J. Med. 335: 462-467.

11. Tepper, J., M.J. Krasna, D. Niedzwiecki, et al. 2008. Phase III trial of trimodality therapy with cisplatin, fluorouracil, radiotherapy, and surgery compared with surgery alone for esophageal cancer: CALGB 9781. J. Clin. Oncol. 26: 10861092.

12. Medical Research Council Oesophageal Cancer Working Group. 2002. Surgical resection with or without preoperative chemotherapy in oesophageal cancer: a randomised controlled trial. Lancet 359: 1727-1733.

13. Stahl, M., M.K. Walz, M. Stuschke, et al. 2009. Phase III comparison of preoperative chemotherapy compared with chemoradiotherapy in patients with locally advanced adenocarcinoma of the esophagogastric junction. J. Clin. Oncol. 27: $851-856$.

14. Illig, R., E. Kleiser, T. Kiesslich \& D. Neureiter. 2013. GERD-Barrett-adenocarcinoma: do we have suitable prognostic and predictive molecular markers? Gastroenterol. Res. Pract. [epub ahead of print] http://dx.doi.org/10.1155/ 2013/643084.

15. Dulak, A.M., P. Stojanov, S. Peng, et al. 2013. Exome and whole-genome sequencing of esophageal adenocarcinoma identified recurrent driver events and mutational complexity. Nat. Genet. 45: 478-486.

16. Agrawal, N., Y. Jiao, C. Bettegowda, et al. 2012. Comparative genomic analysis of esophageal adenocarcinoma and squamous cell carcinoma. Cancer Discov. 2: 899-905.

17. Ayyappan, S., D. Prabhakar \& S. Sharma. 2013. Epidermal growth factor receptor (EGFR)-targeted therapies in esophagogastric cancer. Anticancer Res. 33: 4139-4156.

18. Bang, Y.J., E. Van Cutsem, A. Feyereislova, et al. 2010. Trastuzumab in combination with chemotherapy versus chemotherapy alone for treatment of HER2-positive advanced gastric or gastro-oesophageal junction cancer (ToGA): a phase 3, open-label, randomized controlled trial. Lancet 376: 687-697.

19. Bettstetter, M., S. Berezowska, G. Keller, et al. 2013. Epidermal growth factor receptor, phosphatidylinositol-3-kinase catalytic subunit/PTEN and KRAS/NRS/BRAF in primary resection esophageal adenocarcinomas: loss of PTEN is associated with worse clinical outcome. Hum. Pathol. 44: 829836.

20. Eto, K., M. Iwatsuki, M. Watanabe, et al. 2014. The microRNA-21/PTEN pathway regulates the sensitivity of HER2-positive gastric cancer cells to Trastuzumab. Ann. Surg. Oncol. 21: 343-350.

21. Farris, A.B., 3rd, E.G. Demicco, L.P. Le, et al. 2011. Clinicopathologic and molecular profiles of microsatellite unstable
Barrett esophagus-associated adenocarcinoma. Am. J. Surg. Pathol. 35: 647-655.

22. Xie, L.X., T.T. Zhai, L.P. Yang, et al. 2013. Lymphangiogenesis and prognostic significance of vascular endothelial growth factor $\mathrm{C}$ in gastro-esophageal junction adenocarcinoma. Int. J. Exp. Pathol. 94: 39-46.

23. Mann, B., M. Madera, I. Klouckova, et al. 2010. A quantitative investigation of fucosylated serum glycoproteins with application to esophageal adenocarcinoma. Electrophoresis 31: 1833-1841.

24. Sarkaria, I.S., N.P. Rizk, M.S. Bains, et al. 2009. Posttreatment endoscopic biopsy is a poor-predictor of pathologic response in patients undergoing chemoradiation therapy for esophageal cancer. Ann. Surg. 249: 764-767.

25. Schneider, P.M., R. Metzger, H. Schaefer, et al. 2008. Response evaluation by endoscopy, rebiopsy, and endoscopic ultrasound does not accurately predict histopathologic regression after neoadjuvant chemoradiation for esophageal cancer. Ann. Surg. 248:902-908.

26. Swisher, S.G., M. Maish, J.J. Erasmus, et al. 2004. Utility of PET, CT, and EUS to identify pathologic responders in esophageal cancer. Ann. Thorac. Surg. 78: 1152-1160; discussion 1152-60.

27. Griffin, J.M., C.E. Reed \& C.E. Denlinger. 2012. Utility of restaging endoscopic ultrasound after neoadjuvant therapy for esophageal cancer. Ann. Thorac. Surg. 93: 1855-1859; discussion 1860.

28. Westerterp, M., H.L. van Westreenen, J.B. Reitsma, et al. 2005. Esophageal cancer: CT, endoscopic US, and FDG PET for assessment of response to neoadjuvant therapysystematic review. Radiology 236: 841-851.

29. van Heijl, M., S.S. Phoa, M.I. van Berge Henegouwen, et al. 2011. Accuracy and reproducibility of 3D-CT measurements for early response assessment of chemoradiotherapy in patients with oesophageal cancer. Eur. J. Surg. Oncol. 37: 10641071.

30. Kwee, R.M. 2010. Prediction of tumor response to neoadjuvant therapy in patients with esophageal cancer with use of 18F FDG PET: a systematic review. Radiology 254: 707717.

31. Lambrecht, M., V. Vandecaveye, F. De Keyzer, et al. 2012. Value of diffusion-weighted magnetic resonance imaging for prediction and early assessment of response to neoadjuvant radiochemotherapy in rectal cancer: preliminary results. Int. J. Radiat. Oncol. Biol. Phys. 82: 863-870.

32. van Rossum, P.S., R. van Hillegersberg, M. Lever, et al. 2013. Imaging strategies in the management of oesophageal cancer: what's the role of MRI? Eur Radiol. 23: 1753-1765.

33. Siewert, J.R., F. Lordick, K. Ott, et al. 2007. Induction chemotherapy in Barrett cancer - Influence on surgical risk ond outcome. Ann. Surg. 246: 624-631.

34. Chang, F., H. Deere, U. Mahadeva \& S. George. 2008. Histopathologic examination and reporting of esophageal carcinomas following preoperative neoadjuvant therapy. Am. J. Clin. Pathol. 129: 252-262.

35. Mandard, A.M., F. Dalibard, J.C. Mandard, et al. 1994. Pathological assessment of tumor-regression after preoperative chemoradiotherapy of esophageal-carcinoma. Cancer 73, 2680-2686. 
36. Langer, R., K. Ott, M. Feith, et al. 2009. Prognostic significance of histopathological tumor regression after neoadjuvant chemotherapy in esophageal adenocarcinomas. Modern Pathol. 22: 1555-1563.

37. Swisher, S.G., W. Hofstetter, T.T. Wu, et al. 2005. Proposed revision of the esophageal cancer staging system to accommodate pathologic response $(\mathrm{pP})$ following preoperative chemoradiation (CRT). Ann. Surg. 241: 810-820.

38. Chirieac, L.R., S.G. Swisher, J.A. Ajani, et al. 2005. Posttherapy pathologic stage predicts survival in patients with esophageal carcinoma receiving preoperative chemoradiation. Cancer 103: 1347-1355.

39. Takebayashi, Y., S. Akiyama, S. Natsugoe, et al. 1998. The expression of multidrug resistance protein in human gastrointestinal tract carcinomas. Cancer 82: 661-666.

40. Aloia, T.A., D.H. Harpole, Jr., C.E. Reed, et al. 2001. Tumor marker expression is predictive of survival in patients with esophageal cancer. Ann. Thorac. Surg. 72: 859-866.

41. Langer, R., K. Ott, M. Feith, et al. 2010 High pretherapeutic thymidylate synthetase and MRP-1 protein levels are associated with nonresponse to neoadjuvant chemotherapy in oesophageal adenocarcinoma patients. J. Surg. Oncol. 102: 503-508.

42. Gonzalez, V.M., M.A. Fuertes, C. Alonso \& J.M. Perez. 2001. Is cisplatin-induced cell death always produced by apoptosis? Mol. Pharmacol. 59: 657-663.

43. Bogush, T.A., T.N. Zabotina, E.A. Bogush, et al. 2003. New approach to vital analysis of functional activity in ABC transporters (markers for multidrug resistance) in solid tumors by the method of flow cytofluorometry. Bull. Exp. Biol. Med. 135: 482-488.

44. Bogush, T.A., A.V. Konukhova, A.B. Ravcheeva, et al. 2003. Inhibition of ABC-transporter(s)' function in non-small cell lung cancer cells by platinum drugs. Antibiot. Khimioter. 48: $11-15$.

45. Brücher, B.L.D.M., A. Bilchik, A. Nissan, et al. 2012. Tumor response criteria: are they appropriate ? Future Oncol. 8: 903-906.

46. Miller, A.B., B. Hoogstraten, M. Staquet \& A. Winkler. 1981. Reporting results of cancer treatment. Cancer 47: 207-214.

47. Gurland, J. \& R.O. Johnson. 1966. Case for using only maximum diameter in measuring tumors. Cancer Chemother. Rep. 50: 119-124.

48. Moertel, C.G. \& J.A. Hanley. 1976. Hanley. The effect of measuring error on the results of therapeutic trials in advanced cancer. Cancer 38: 388-394.

49. Breast Cancer Task Force Treatment Committee, 1977. National Cancer Institute: report from the combination chemotherapy trials working group. U.S. Department of Health, Education and Welfare. DHEW Publication No. (NIH) 77-1192.

50. Therasse, P., S.G. Arbuck, E.A. Eisenhauer, et al. 2000. New guidelines to evaluate the response to treatment in solid tumors. European Organization for Research and Treatment of Cancer, National Cancer Institute of the United States,
National Cancer Institute of Canada. J. Natl. Cancer Inst. 92: 205-216.

51. Brücher, B.L.D.M., H.J. Stein, M. Werner \& J.R. Siewert. 2001. Lymphatic vessel invasion is an independent prognostic factor in patients with a primary resected tumor with esophageal squamous cell carcinoma. Cancer 92: 2228-2233.

52. Brücher, B.L.D.M., K. Becker, F. Lordick, et al. 2006. The clinical impact of histopathologic response assessment by residual tumor cell quantification in esophageal squamous cell carcinomas. Cancer 106: 2119-2127.

53. Brücher, B.L.D.M., S. Swisher, A. Königsrainer, et al. 2009. Response to preoperative therapy in upper gastrointestinal cancers. Ann. Surg. Oncol. 16: 878-886.

54. Dikken, J., V. Lemmens, et al. 2012. Increased incidence and survival for oesophageal cancer but not for gastric cardia cancer in the Netherlands. Eur. J. Cancer 48: 1624-1632.

55. Smit, J., G. Sahin, et al. 2013. Different recurrence pattern after neoadjuvant chemoradiotherapy compared to surgery alone in esophageal cancer patients. Ann. Surg. Oncol. 20: 4008-4015.

56. Blom, R., W. Schreurs, et al. 2011. The value of postneoadjuvant therapy PET-CT in the detection of interval metastases in esophageal carcinoma. Eur. J. Surg. Oncol. 37: 774-778.

57. Bruzzi, J., S. Swisher, et al. 2007. Detection of interval distant metastases: clinical utility of integrated CT-PET imaging in patients with esophageal carcinoma after neoadjuvant therapy. Cancer 109: 125-134.

58. Cerfolio, R., A. Bryant, et al. 2005. The accuracy of endoscopic ultrasonography with fine-needle aspiration, integrated positron emission tomography with computed tomography, and computed tomography in restaging patients with esophageal cancer after neoadjuvantchemoradiotherapy. J. Thorac. Cardiovasc. Surg. 129: 1232-1241.

59. van Heijl, M., J.M. Omloo, M.I. van Berge Henegouwen, et al. 2011. Fluorodeoxyglucose positron emission tomography for evaluating early response during neoadjuvant chemoradiotherapy in patients with potentially curable esophageal cancer. Ann. Surg. 253: 56-63.

60. Vliegen, R. et al. Assessment of tumor response to neoadjuvante therapy in patients with esophageal cancer with use of diffusion-weighted MRI: initial experience. Unpublished data.

61. van Baardwijk, A., C. Dooms, et al. 2007. The maximum uptake of (18)F-deoxyglucose on positron emission tomography scan correlates with survival, hypoxia inducible factor-1 alpha and GLUT-1 in non-small cell lung cancer. Eur. J. Cancer 43: 1392-1398.

62. van Loon, J., M. Janssen, et al. 2010. PET imaging of hypoxia using $\left({ }^{18} \mathrm{~F}\right) \mathrm{HX} 4$ : a phase I trial. Eur. J. Nucl. Med. Mol. Imaging 37: 1663-1668.

63. Shapiro, J., F.J. ten Kate, P. van Hagen, K. Biermann, B.P. Wijnhoven \& J.J. van Lanschot. 2013. Residual esophageal cancer after neoadjuvant chemoradiotherapy frequently involves the mucosa and submucosa. Ann. Surg. 258: 678-688. 